Pacific

Journal of

Mathematics

\title{
THE FORGETFUL MAP IN RATIONAL $K$-THEORY
}

WILLIAM GRAHAM 


\title{
THE FORGETFUL MAP IN RATIONAL $K$-THEORY
}

\author{
WILLIAM GRAHAM
}

Let $G$ be a connected reductive algebraic group acting on a scheme $X$. Let $R(G)$ denote the representation ring of $G$, and $I \subset R(G)$ the ideal of virtual representations of $\operatorname{rank} 0$. Let $G(X)$ (respectively, $G(G, X)$ ) denote the Grothendieck group of coherent sheaves (respectively, $G$-equivariant coherent sheaves) on $X$. Merkurjev proved that if $\pi_{1}(G)$ is torsion-free, then the forgetful map $G(G, X) \rightarrow G(X)$ induces an isomorphism

$$
G(G, X) / I G(G, X) \rightarrow G(X) .
$$

Although this map need not be an isomorphism if $\pi_{1}(G)$ has torsion, we prove that without the assumption on $\pi_{1}(G)$, the map $G(G, X) / I G(G, X) \otimes$ $\mathbb{Q} \rightarrow G(X) \otimes \mathbb{Q}$ is an isomorphism.

\section{Introduction}

Let $G$ be a connected reductive algebraic group acting on a scheme $X$. The $G$ equivariant coherent sheaves on $X$ are central to the study of $X$. These sheaves often have computable invariants, since the group action allows the use of tools such as localization theorems. Also, equivariant sheaves are an important source of sheaves on quotients by group actions, since if a quotient $X \rightarrow Y$ exists, then the sheaf of invariant sections of an equivariant sheaf on $X$ is a coherent sheaf on $Y$. It is natural to ask which coherent sheaves on $X$ admit $G$-actions. One positive result is due to Mumford, who proved that if $G$ is connected and $X$ is normal, and $L$ is any invertible sheaf on $X$, then some power of $L$ is $G$-linearizable [Mumford et al. 1994, Corollary 1.6]. On the other hand, it is easy to find examples of coherent sheaves which do not admit $G$-actions. For example, PGL(2) acts on $\mathbb{P}^{1}$ but the sheaf $\mathscr{O}_{\mathbb{p l}^{1}}(1)$ does not admit an action of PGL(2); see [Mumford et al. 1994, p. 33].

Merkurjev [1997] proved that from the point of view of $K$-theory, there is no obstruction to equivariance, as long as the fundamental group of $G$ is torsionfree. Let $G(X)$ and $G(G, X)$ denote the Grothendieck groups of, respectively, coherent sheaves and $G$-equivariant coherent sheaves on $X$. There is a forgetful

MSC2000: 19E08, 18F30.

Keywords: $K$-theory, equivariant, equivariant $K$-theory, Riemann-Roch.

The author was supported by the National Science Foundation. 
map $G(G, X) \rightarrow G(X)$. Let $R=R(G)$ denote the representation ring of $G$, and $I \subset R$ the augmentation ideal, that is, the ideal of virtual representations of rank 0 . The Grothendieck group $G(G, X)$ is an $R$-module. Merkurjev showed that if $\pi_{1}(G)$ is torsion-free, then the forgetful map induces an isomorphism

$$
G(G, X) / I G(G, X) \rightarrow G(X) .
$$

If $\pi_{1}(G)$ is not torsion-free, this map can fail to be an isomorphism. For example, the fundamental group of PGL(2) is $\mathbb{Z} / 2 \mathbb{Z}$, and the class $v=\left[\mathbb{O}_{\mathbb{P}^{1}}(1)\right] \in G\left(\mathbb{P}^{1}\right)$ is not in the image of $G\left(\operatorname{PGL}(2), \mathbb{P}^{1}\right)$. However, if we tensor with $\mathbb{Q}$, this class is in the image. Indeed, $G\left(\mathbb{P}^{1}\right)=\mathbb{Z}[v] /\left\langle(v-1)^{2}\right\rangle$, so after tensoring with $\mathbb{Q}$, we have $v=\frac{1}{2}\left(v^{2}+1\right)$. This element is in the image of the forgetful map since $v^{2}$ is the class of $O_{\mathbb{P}^{1}}(2)$, which has a $G$-action.

This phenomenon holds more generally:

Theorem 1.1. Let $G$ be a connected reductive algebraic group acting on a scheme $X$. The forgetful map $G(G, X) \rightarrow G(X)$ induces an isomorphism

$$
G(G, X) / I G(G, X) \otimes \mathbb{Q} \rightarrow G(X) \otimes \mathbb{Q} .
$$

Hence the map $G(G, X) \otimes \mathbb{Q} \rightarrow G(X) \otimes \mathbb{Q}$ is surjective.

Merkurjev proves his theorem by using a spectral sequence relating equivariant and ordinary $K$-theory. The approach taken in this paper is different, and makes use of Brion's analogue of Theorem 1.1 for Chow groups, along with the equivariant Riemann-Roch theorem proved by Edidin and the author. This use of RiemannRoch explains the rational coefficients in the statement of our theorem.

We remark that Theorem 1.1 remains true even if $G$ is not reductive, provided that $G$ has a Levi factor $L$ (which is automatic in characteristic 0 ), since then the forgetful maps from $G$-equivariant $K$-theory and Chow groups to the corresponding $L$-equivariant groups are isomorphisms. Also, we expect that a topological version of Theorem 1.1 holds for equivariantly formal spaces (since for these spaces the map from equivariant cohomology to ordinary cohomology is surjective). Finally, the completion theorem of [Edidin and Graham 2007] should have implications in this setting.

Conventions. We work over an algebraically closed field $k$. We will assume our schemes admit closed equivariant embeddings into smooth schemes. This assumption has the following consequences, which we will use in the paper. First, it ensures that the mixed spaces we use exist as schemes; see [Edidin and Graham 1998, Proposition 23]. Second, it allows us to make use of functorial properties of Riemann-Roch (see [Fulton 1984, Theorem 18.3(4)]). Third, it implies that the $G$ actions are locally linear - that is, the schemes on which $G$ acts can be covered by $G$-invariant quasiprojective open subsets. Since this holds for normal schemes, it 
also holds for closed subschemes of normal schemes. Brion's results are proved for locally linear actions over algebraically closed fields, so we can apply his results.

We remark that Merkurjev does not assume that $k$ is algebraically closed; if $k$ is not algebraically closed then he assumes that $G$ is split.

\section{Equivariant $K$-theory, Chow groups, and Riemann-Roch}

In this section we recall some basic facts about $K$-theory, Chow groups, and Riemann-Roch, in the equivariant and nonequivariant settings. We prove a result comparing topologies on equivariant Chow groups, and also prove a compatibility result between Riemann-Roch and forgetful maps. Both of these results are used in the proof of the main theorem. Our main references for equivariant Chow groups and equivariant Riemann-Roch will be [Edidin and Graham 1998] and [Edidin and Graham 2000], where more details can be found. If $M$ is an abelian group, we write $M_{\mathbb{Q}}=M \otimes_{\mathbb{Z}} \mathbb{Q}$. Because we want to index Chow groups by codimension, we will assume all schemes and algebraic spaces considered are equidimensional; our results are valid without this assumption, but we would have to index Chow groups by dimension.

We begin with some definitions. Let $G$ be a linear algebraic group acting on an algebraic space $X$. Let $G(G, X)$ (respectively, $G(X))$ denote the Grothendieck group of $G$-equivariant coherent sheaves (respectively, coherent sheaves). There is a forgetful map

$$
\text { For : } G(G, X) \rightarrow G(X)
$$

which takes the class of a $G$-equivariant coherent sheaf to the class of the same sheaf, viewed nonequivariantly. If we need to keep track of the space involved, we will denote this by For $_{X}$. Note that $G(G, X)$ is a module for the representation ring $R=R(G)$ of $G$. Let $I \subset R$ denote the augmentation ideal (the ideal of virtual representations of rank 0). Let $G \widehat{(G, X)})_{\mathbb{Q}}$ denote the $I$-adic completion of $G(G, X)_{\mathbb{Q}}$ (not the tensor product with $\mathbb{Q}$ of the $I$-adic completion of $G(G, X)$ ).

Let $C H^{i}(X)$ denote the codimension $i$ Chow group of $X$; if $X$ has pure dimension $d$, then $C H^{i}(X)=A_{d-i}(X)$. Write $C H^{*}(X)=\bigoplus_{i} C H^{i}(X)$. Similarly, let $C H_{G}^{i}(X)=A_{d-i}^{G}(X)$ denote the "codimension $i$ " equivariant Chow group of $X$, and $C H_{G}^{*}(X)=\bigoplus C H_{G}^{i}(X)$. By definition, if $V$ is a representation of $G$ and $U$ an open subset of $V$ on which $G$ acts freely, then $C_{G}^{i}(X)=C H^{i}((X \times U) / G)$. This definition is independent of the choice of $V$ and $U$ (see [Edidin and Graham 1998]). We will denote the mixed space $(X \times U) / G$ by $X \times{ }^{G} U$ or $X_{G}$. Now, $X$ is embedded in $X_{G}$ as a fiber of the map $X_{G} \rightarrow U / G$, and pullback along this embedding gives a map

$$
\text { For : } \mathrm{CH}_{G}^{i}(X) \rightarrow C H^{i}(X) \text {. }
$$


Note that $C H_{G}^{*}(X)$ is a module for the graded ring $S=C H_{G}^{*}(\mathrm{pt})$. Let $J \subset S$ be the ideal spanned by the homogeneous elements of $S$ of positive degree.

The following proposition is similar to [Edidin and Graham 2000, Proposition 2.1], which dealt with the case where $G$ is a subgroup of the group of upper triangular matrices. The proof is a minor modification of that proof.

Proposition 2.1. Let $G$ be a connected reductive algebraic group acting on a scheme $X$. Let $N=C_{G}^{*}(X)_{\mathbb{Q}}$. The topologies on $N$ induced by the two filtrations $\left\{J^{n} N\right\}$ and $\left\{\bigoplus_{i \geq n} N^{i}\right\}$ coincide.

Proof. We must show two things. First, given any $n$, there exists an $r$ such that $J^{r} N \subset \bigoplus_{i \geq n} N^{i}$. For this we may take $n=r$, since $N$ is nonnegatively graded and $J N^{i} \subseteq N^{i+1}$. Second, given any $n$, there exists an $r$ such that $\bigoplus_{i \geq r} N^{i} \subseteq J^{n} N$. Indeed, Brion proved that $N / J N \simeq C H^{*}(X)_{\mathbb{Q}}$. Thus, $N / J N$ is 0 in degrees greater than $d=\operatorname{dim} X$, so $N^{p}=J N^{p-1}$ for $p>d$. Thus, for $p \geq n+d$, we have $N^{p}=J^{n} N^{p-n}$, so for $r=n+d$, we have $\bigoplus_{i \geq r} N^{i} \subseteq J^{n} N$, as desired.

Corollary 2.2. Let $G$ be a connected reductive algebraic group acting on a scheme $X$. Then the J-adic completion of $\mathrm{CH}_{G}^{*}(X)_{\mathbb{Q}}$ is isomorphic to the direct product $\prod_{i=0}^{\infty} C H_{G}^{i}(X)_{\mathbb{Q}}$.

Proof. Since the completion of $\mathrm{CH}_{G}^{*}(X)_{\mathbb{Q}}$ with respect to the topology induced by the second filtration above is the direct product $\prod_{i=0}^{\infty} C H_{G}^{i}(X)_{\mathbb{Q}}$, this follows from the preceding proposition.

Edidin and Graham [2000] constructed an equivariant Riemann-Roch map

$$
\tau_{X}^{G}: G(G, X) \rightarrow \prod_{i} C H_{G}^{i}(X)_{\mathbb{Q}},
$$

with the same functorial properties as the nonequivariant Riemann-Roch map

$$
\tau_{X}: G(X) \rightarrow C H^{*}(X)_{\mathbb{Q}}
$$

of [Fulton 1984]. The equivariant Riemann-Roch map induces an isomorphism

$$
\left.\hat{\tau}_{X}^{G}: G \widehat{(G, X)}\right)_{\mathbb{Q}} \rightarrow \prod_{i} C H_{G}^{i}(X)_{\mathbb{Q}} .
$$

(In [Edidin and Graham 2000], $\hat{\tau}_{X}^{G}$ was denoted simply by $\tau_{X}^{G}$.) Also, there is an equivariant Chern character map $\operatorname{ch}_{G}: R \rightarrow S$ which takes $I$ to $J$ and induces an isomorphism of the $I$-adic completion $\hat{R}$ of $R$ with the $J$-adic completion $\hat{S}$ of $S$. Using $\operatorname{ch}_{G}$ to identify $\hat{R}$ with $\hat{S}$, the functorial properties of $\hat{\tau}_{X}^{G}$ (see [Edidin and Graham 2000, Theorem 3.1(c)]) imply that is an isomorphism of $\hat{R}=\hat{S}$-modules.

The forgetful maps in $K$-theory and Chow groups are compatible with the Riemann-Roch maps, by the following proposition. Let

$$
\tau_{X}^{G, i}: G(G, X) \rightarrow C H_{G}^{i}(X)_{\mathbb{Q}} \quad\left(\text { respectively, } \tau_{X}^{i}: G(X) \rightarrow C H^{i}(X)_{\mathbb{Q}}\right)
$$


be the composition of the map $\tau_{X}^{G}$ (respectively, $\tau_{X}$ ) with the projection to the component of degree $i$.

Proposition 2.3. Let $G$ be a linear algebraic group acting on a scheme $X$. The following diagram commutes:

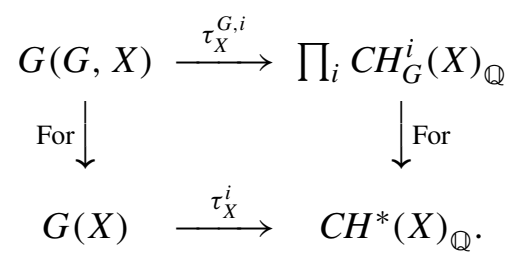

Proof. This can be proved using a change of groups argument along the lines of [Edidin and Graham 2000, Lemma 4.3]. Here we give a more direct proof. Let $V$ be a representation of $G$ and $U$ an open subset of $V$ on which $G$ acts freely, such that the codimension of $V-U$ is greater than $i$. By definition,

$$
C H_{G}^{i}(X)=C H^{i}\left(X_{G}\right),
$$

where $X_{G}=X \times{ }^{G} U$. Let

$$
\pi: X \times U \rightarrow X
$$

denote the projection, and let

$$
q: X \times U \rightarrow X_{G}
$$

denote the quotient map. If $\mathscr{F}$ is a coherent sheaf on $X_{G}$, then the pullback sheaf $q^{*} \mathscr{F}_{F}$ on $X \times U$ has a natural $G$-action. The assignment $\mathscr{F}_{F} \rightarrow q^{*} \mathscr{F}_{F}$ gives an equivalence of categories between the category of coherent sheaves on $X \times{ }^{G} U$ and the category of $G$-equivariant coherent sheaves on $X \times U$ (this follows from Thomason's work in [1987]; see [Edidin and Graham 2000] for a discussion). This equivalence yields an isomorphism $G\left(X_{G}\right) \rightarrow G(G, X \times U)$, denoted by $q^{*}$.

Let $u \in U$ and let $v \in U / G$ be the image of $u$. Let $j: X \rightarrow X \times U$ take $X$ to $X \times\{u\}$, and let $k=q \circ j: X \rightarrow X_{G}$. Then $k$ is the inclusion of $X$ as the fiber of $X_{G} \rightarrow U / G$ over $v$. The normal bundle $N_{k}$ to $k$ is pulled back from the normal bundle to the inclusion of $v$ in $U / G$, so $N_{k}$ is trivial, and hence by [Fulton 1984, Theorem 18.3],

$$
\tau_{X} \circ k^{!}=k^{*} \circ \tau_{X_{G}}
$$

as maps $G\left(X_{G}\right) \rightarrow C H^{*}(X)_{\mathbb{Q}}$.

Let $\mathscr{V}$ denote the vector bundle $X \times{ }^{G}(U \times V) \rightarrow X_{G}$. Define

$$
\rho_{U}: G\left(X_{G}\right) \rightarrow C H^{*}\left(X_{G}\right)
$$


by

$$
\rho_{U}(\beta)=\frac{\tau_{X_{G}}(\beta)}{\operatorname{Td}(\mathscr{V})} .
$$

Let $\rho_{U}^{i}$ be the composition of $\rho_{U}$ with the projection onto the $i$-th component. Then by the definition of the equivariant Riemann-Roch map (see [Edidin and Graham 2000]), $\tau_{X}^{G, i}$ is the top row of the following diagram:

$$
\begin{aligned}
& G(G, X) \stackrel{\pi^{!}}{\longrightarrow} G(G, X \times U) \stackrel{\left(q^{*}\right)^{-1}}{\longrightarrow} G\left(X_{G}\right) \stackrel{\rho_{U}^{i}}{\longrightarrow} C H^{i}\left(X_{G}\right)=C H_{G}^{i}(X) \\
& k^{!} \\
& G(X) \stackrel{\tau_{X}^{i}}{\longrightarrow} C H^{*}(X) .
\end{aligned}
$$

Here $\pi !$ is the flat pullback in equivariant $K$-theory; if $\mathscr{E}$ is an equivariant coherent sheaf on $X$ then $\pi^{!}[\mathscr{E}]=\left[\pi^{* \mathscr{E}}\right]$, where $\pi^{* \mathscr{E}}$ is the pullback of the sheaf $\mathscr{E}$. Also, $k^{!}$ and $k^{*}$ are the Gysin morphisms associated to the regular embedding $k$ (see [Fulton 1984]). The pullback along $k$ of the vector bundle $\mathscr{V}$ is trivial, so $k^{*}(\operatorname{Td}(\mathscr{V}))=1$. Hence (1) and (2) imply that the diagram commutes. To complete the proof of the proposition, it suffices to show that

$$
k^{!} \circ\left(q^{*}\right)^{-1} \circ \pi^{!}=\text {For }_{X}
$$

as maps $G(G, X) \rightarrow G(X)$. Now, $k^{!}=j^{!} q^{!}$, so the left hand side of (3) is

$$
j ! q^{!}\left(q^{*}\right)^{-1} \pi !
$$

By definition, $\left(q^{*}\right)^{-1}$ takes the class of an equivariant coherent sheaf $\mathscr{F}$ to the class of a nonequivariant sheaf $\mathscr{E}$ with $q^{* \mathscr{E}}=\mathscr{F}$. On the other hand, $q^{!}[\mathscr{E}]$ is the class of $q^{* \mathscr{E}}$ (viewed as a nonequivariant coherent sheaf) in $G(X \times U)$. Thus, the composition $q^{!}\left(q^{*}\right)^{-1}$ is $\operatorname{For}_{X \times U}: G(G, X \times U) \rightarrow G(X \times U)$. Since the forgetful map commutes with flat pullback, (4) equals

$$
j ! \circ \operatorname{For}_{X \times U} \circ \pi^{!}=j^{!} \pi ! \circ \operatorname{For}_{X}=(\pi \circ j)^{!} \circ \operatorname{For}_{X}=\operatorname{For}_{X},
$$

as desired.

\section{Completions}

The purpose of this section is to prove a simple result (Lemma 3.1) about completions. This lemma is certainly known (compare [Bourbaki 1972, p. 247] for finitely generated modules), but because of a lack of a reference for nonfinitely generated modules, a proof is included.

Let $R$ be a Noetherian ring and $I$ an ideal of $R$. Let $\hat{M}$ denote the $I$-adic completion of the $R$-module $M$. We view $\hat{M}$ as the set of coherent sequences 
$\left(m_{1}, m_{2}, \ldots\right)$; here $m_{k} \in M / I^{k} M$, and coherent means that for all $k$, the natural map $M / I^{k+1} M \rightarrow M / I^{k} M$ takes $m_{k+1}$ to $m_{k}$. Since $\hat{I}=I \hat{R}$ [Atiyah and Macdonald 1969, Proposition 10.15], we have $\hat{I} \hat{M}=I \hat{R} \hat{M}=I \hat{M}$. The composition $M \rightarrow$ $\hat{M} \rightarrow M / I \hat{M}$ induces a map $f: M / I M \rightarrow \hat{M} / I \hat{M}$.

Lemma 3.1. Let $R$ be a Noetherian ring and $I$ an ideal of $R$. For any $R$-module $M$, the map $f: M / I M \rightarrow \hat{M} / I \hat{M}$ is an isomorphism.

Proof. The exact sequence $0 \rightarrow I M \rightarrow M \stackrel{\pi}{\rightarrow} M / I M \rightarrow 0$ yields an exact sequence of completions

$$
0 \rightarrow \widehat{I M} \rightarrow \hat{M} \stackrel{\hat{\pi}}{\rightarrow} \widehat{M / I M}=M / I M \rightarrow 0
$$

(see [Atiyah and Macdonald 1969, Cor. 10.3, 10.4]). The map $\hat{\pi}: \hat{M} \rightarrow M / I M$ takes the coherent sequence $\mu=\left(m_{1}, m_{2}, \ldots\right)$ to $m_{1}$. We claim that the subspaces $\widehat{I M}$ and $I \hat{M}$ of $\hat{M}$ are equal. This suffices, for then the map $p: \hat{M} / I \hat{M} \rightarrow M / I M$ (induced from $\hat{\pi}$ ) is an isomorphism. Indeed, the map $f: M / I M \rightarrow \hat{M} / I \hat{M}$ is induced from the map $M \rightarrow \hat{M} / I \hat{M}$ taking $m$ to $\left(m_{1}, m_{2}, \ldots\right)$, where we set $m_{k}=m \bmod I^{k} M$. Since $p \circ f$ is the identity map of $M / I M$, the claim implies that $f$ is an isomorphism.

It remains to prove the claim. As noted above, $\operatorname{ker} \hat{\pi}=\widehat{I M}$. Clearly $I \hat{M} \subseteq \operatorname{ker} \hat{\pi}$, so we must show the reverse inclusion.

Given an element $\mu=\left(m_{1}, m_{2}, \ldots\right) \in \hat{M}$, let $p_{k}(\mu)=m_{k} \in M / I^{k} M$. Let $a_{1}, \ldots, a_{n}$ generate $I$. Suppose that $\mu \in \operatorname{ker} \hat{\pi}$. We want to show that $\mu \in I \hat{M}$. Now, $p_{1}(\mu)=0$, and $p_{2}(\mu) \in I M / I^{2} M$. Let $\mu^{1}, \ldots, \mu^{n}$ be elements of $M$ such that $\sum a_{i} \mu^{i} \bmod I^{2} M=p_{2}(\mu)$. Let $\hat{\mu}^{i}$ be the image of $\mu^{i}$ under $M \rightarrow \hat{M}$, and let

$$
\mu(2)=\mu-\sum a_{i} \hat{\mu}^{i} .
$$

Then $p_{1}(\mu(2))=p_{2}(\mu(2))=0$, so $p_{3}(\mu(2)) \in I^{2} M / I^{3} M$. Let $\mu^{i j}$ be elements of $M$ such that $\sum a_{i} a_{j} \mu^{i j} \bmod I^{3} M=p_{3}(\mu(2))$. Let $\hat{\mu}^{i j}$ be the image of $\mu^{i j}$ under $M \rightarrow \hat{M}$, and let

$$
\mu(3)=\mu(2)-\sum a_{i} a_{j} \hat{\mu}^{i j} .
$$

Then $p_{i}(\mu(3))=0$ for $i \leq 3$. Proceeding inductively, suppose we have $\mu(k) \in \hat{M}$ with $p_{i}(\mu(k))=0$ for $i \leq k$. Then we can find elements $\mu^{J} \in M$, where $J$ runs over the collection of all $k$-element multisets with elements in $\{1,2, \ldots, n\}$, such that if we define

$$
\mu(k+1)=\mu(k)-\sum_{|J|=k} a^{J} \hat{\mu}^{J},
$$

then we have $p_{j}(\mu(k+1))=0$ for $j \leq k+1$. (Here $|J|$ is the number of elements in $J$, counted with multiplicity; $a^{J}=\prod_{j \in J} a_{j}$, where each $a_{j}$ occurs with its 
multiplicity in $J$; and $\hat{\mu}^{J}$ is the image of $\mu^{J}$ under $M \rightarrow \hat{M}$.) Then

$$
\mu=\sum_{k} \sum_{|J|=k} a^{J} \mu^{J}
$$

that is, the right hand side converges to the element $\mu \in \hat{M}$. Let $S_{i}$ be the collection of multisets whose smallest element is $i$. We can rewrite the preceding equation as

$$
\mu=a_{1} \sum_{J \in S_{1}} a^{J-\{1\}} \mu^{J}+a_{2} \sum_{J \in S_{2}} a^{J-\{2\}} \mu^{J}+\cdots+a_{n} \sum_{J \in S_{n}} a^{J-\{n\}} \mu^{J} .
$$

Each of the series $\sum_{J \in S_{i}} a^{J-\{i\}} \mu^{J}$ converges to an element of $\hat{M}$, so we conclude that $\mu \in I \hat{M}$, as desired.

Remark 3.2. In the proof of the lemma, the claim that $\widehat{I M}=I \hat{M}$ admits a simpler proof if $M$ is finitely generated. Indeed, by [Atiyah and Macdonald 1969, Proposition 10.13], in this case the horizontal maps in the following commutative diagram are isomorphisms:

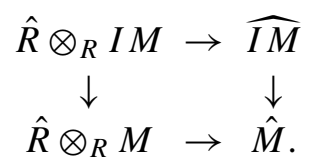

The image in $M$ of $\hat{R} \otimes_{R} I M$ under the upper (respectively, lower) composition is $\widehat{I M}$ (respectively, $I \hat{M}$ ), so $\widehat{I M}=I \hat{M}$ as desired.

\section{Proof of Theorem 1.1}

In this section we work with rational coefficients and tensor all Grothendieck groups and Chow groups with $\mathbb{Q}$. For simplicity we will omit this from the notation and simply write, for example, $G(G, X)$ for $G(G, X)_{\mathbb{Q}}$, or $R$ for $R_{\mathbb{Q}}$. If $M$ is an $R$ module we will write $M / I$ for $M / I M$, and if $N$ is an $S$-module we will write $N / J$ for $N / J N$. Recall that by Corollary 2.2 we can identify the $J$-adic completion of $C H_{G}^{*}(X)$ with the direct product $\prod_{i=0}^{\infty} C H_{G}^{i}(X)$.

By Proposition 2.3, we have a commutative diagram

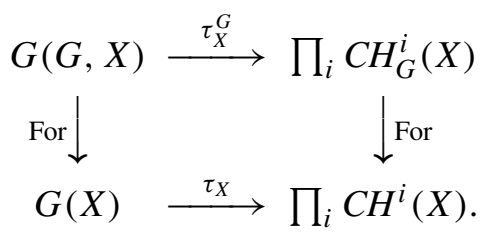

Now, $\tau_{X}^{G}$ takes $I G(G, X)$ to $J \prod C H_{G}^{i}(X)$. Also, the forgetful maps factor as

$$
G(G, X) \rightarrow G(G, X) / I \rightarrow G(X)
$$


and

$$
\prod_{i} C H_{G}^{i}(X) \rightarrow\left(\prod_{i} C H_{G}^{i}(X)\right) / J \rightarrow C H^{*}(X) .
$$

Therefore, we obtain a commutative diagram

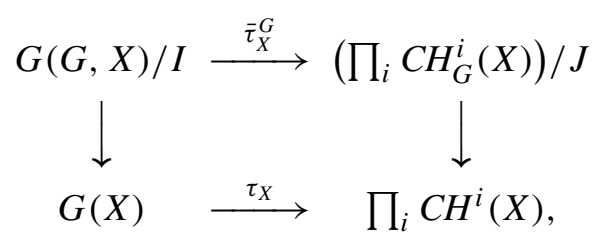

where $\bar{\tau}_{X}^{G}$ is induced from $\tau_{X}^{G}$. The map $\tau_{X}$ is an isomorphism (see [Fulton 1984, Corollary 18.3.2]). We claim that $\bar{\tau}_{X}^{G}$ is as well. Indeed, $\tau_{X}^{G}$ factors as

$$
G(G, X) \rightarrow \widehat{G(G, X)} \stackrel{\hat{\tau}_{X}^{G}}{\rightarrow} \prod_{i} C H_{G}^{i}(X) .
$$

and the map $\hat{\tau}_{X}^{G}$ is an isomorphism. As observed in Section 2, if we use $\operatorname{ch}_{G}$ to identify $\hat{R}$ with $\hat{S}$, then $\hat{\tau}_{X}^{G}$ is an isomorphism of $\hat{R}=\hat{S}$-modules. Hence $\hat{\tau}_{X}^{G}$ induces an isomorphism

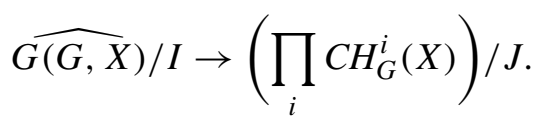

(Here we are using the fact that $\hat{I}=I \hat{R}$, so $\widehat{G(G, X)} / \hat{I}=\widehat{G(G, X)} / I$; similarly, $\left.\left(\prod_{i} C H_{G}^{i}(X)\right) / \hat{J}=\left(\prod_{i} C H_{G}^{i}(X)\right) / J\right)$. We can write $\bar{\tau}_{X}^{G}$ as the composition

$$
G(G, X) / I \rightarrow \widehat{G(G, X)} / I \rightarrow\left(\prod_{i} C H_{G}^{i}(X)\right) / J
$$

Since the second map is an isomorphism, and by Lemma 3.1 the first map is an isomorphism as well, we conclude that $\bar{\tau}_{X}^{G}$ is an isomorphism, proving the claim.

Now, we have a commutative diagram

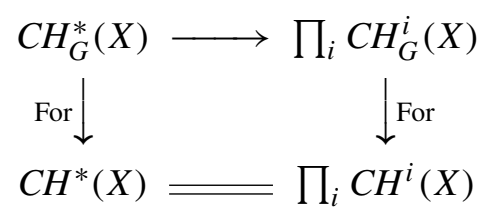

(the bottom equality is because $C H^{i}(X)$ is zero for $i<0$ or $i>\operatorname{dim} X$ ). From this we obtain a commutative diagram

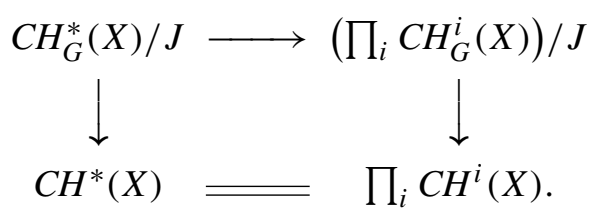


The top map is an isomorphism by Corollary 2.2 and Lemma 3.1, and Brion proved that the left vertical map is an isomorphism. Hence, combining diagrams (5) and (6), we obtain a commutative diagram

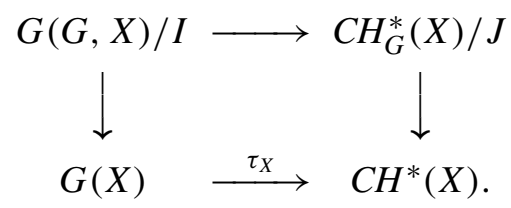

Since the top, bottom, and right vertical maps are isomorphisms, we conclude that the left vertical map is an isomorphism as well. This completes the proof.

Example 4.1. We return to the example of $G=\operatorname{PGL}(2)$ acting on $\mathbb{P}^{1}$, considered in the introduction. Let $B$ denote the stabilizer in $G$ of the point [1:0] and let $T$ denote the maximal torus which is the image of the diagonal matrices in GL(2) under the quotient map GL(2) $\rightarrow \operatorname{PGL}(2)$. Then $\mathbb{P}^{1}$ can be identified with $G / B$, and a standard change of groups argument (see for example [Edidin and Graham 2000, Proposition 3.2]) implies

$$
G(G, G / B)=G(B, \mathrm{pt})=R(B)=R(T) .
$$

Since we are working with rational coefficients, $R(T) \simeq \mathbb{Q}\left[u, u^{-1}\right]$ and this isomorphism can be chosen so that $u$ corresponds to $\left[\mathbb{O}_{\mathbb{P}^{1}}(2)\right]$ in $G\left(G, \mathbb{P}^{1}\right)$. We may view $R(G)$ as the subring $\mathbb{Q}\left[u+u^{-1}\right]$ of $R(T)$; then the ideal $I \subset R(G)$ is generated by $u+u^{-1}-2$, so $G\left(G, \mathbb{P}^{1}\right) / I=\mathbb{Q}\left[u, u^{-1}\right] /\left\langle(u-1)^{2}\right\rangle$. Also, if $v=\left[\mathbb{O}_{\mathbb{P}^{1}}(1)\right] \in G\left(\mathbb{P}^{1}\right)$, then $G\left(\mathbb{P}^{1}\right)=\mathbb{Q}[v] /\left\langle(v-1)^{2}\right\rangle$. The forgetful map $G\left(G, \mathbb{P}^{1}\right) \rightarrow G\left(\mathbb{P}^{1}\right)$ takes $u$ to $v^{2}$, and induces an isomorphism $G\left(G, \mathbb{P}^{1}\right) / I \simeq G\left(\mathbb{P}^{1}\right)$. However, if we were working with integer coefficients, the forgetful map would not be surjective, since in that case $v$ is not in the image.

\section{References}

[Atiyah and Macdonald 1969] M. F. Atiyah and I. G. Macdonald, Introduction to commutative algebra, Addison-Wesley, Reading, MA, 1969. MR 39 \#4129 Zbl 0175.03601

[Bourbaki 1972] N. Bourbaki, Elements of mathematics. Commutative algebra, Hermann, Paris, 1972. Translated from the French. MR 50 \#12997 Zbl 0279.13001

[Edidin and Graham 1998] D. Edidin and W. Graham, "Equivariant intersection theory", Invent. Math. 131:3 (1998), 595-634. MR 99j:14003a Zbl 0940.14003

[Edidin and Graham 2000] D. Edidin and W. Graham, "Riemann-Roch for equivariantChow groups", Duke Math. J. 102:3 (2000), 567-594. MR 2001f:14018 Zbl 0997.14002

[Edidin and Graham 2007] D. Edidin and W. Graham, "Algebraic cycles and completions of equivariant $K$-theory”, preprint, 2007. arXiv math.AG/0702671

[Fulton 1984] W. Fulton, Intersection theory, Ergebnisse der Mathematik (3) 2, Springer, Berlin, 1984. MR 85k:14004 Zbl 0541.14005 
[Merkurjev 1997] A. S. Merkur'ev, "Comparison of the equivariant and the standard $K$-theory of algebraic varieties”, Algebra i Analiz 9:4 (1997), 175-214. MR 99d:19003 Zbl 0897.19004

[Mumford et al. 1994] D. Mumford, J. Fogarty, and F. Kirwan, Geometric invariant theory, 3rd ed., Ergebnisse der Mathematik (2) 34, Springer, Berlin, 1994. MR 95m:14012 Zbl 0797.14004

[Thomason 1987] R. W. Thomason, "Algebraic $K$-theory of group scheme actions", pp. 539-563 in Algebraic topology and algebraic K-theory (Princeton, NJ, 1983), edited by W. Browder, Ann. of Math. Stud. 113, Princeton Univ. Press, Princeton, NJ, 1987. MR 89c:18016 Zbl 0701.19002

Received October 29, 2007.

WILLIAM GRAHAM

DEPARTMENT OF MATHEMATICS

UNIVERSITY OF GEORGIA

Boyd Graduate Studies Research CENTER

ATHENS, GA 30602

UNITED STATES

wag@math.uga.edu 